\title{
Social Translucence as a Theoretical Framework for Sustainable HCI
}

\author{
Mary Barreto, Evangelos Karapanos, and Nuno Nunes \\ Madeira Interactive Technologies Institute, \\ University of Madeira, Caminho da Penteada, \\ Funchal, 9020-105, Madeira, Portugal \\ \{maryluizbarreto, e.karapanos\} @gmail.com, njn@uma.pt
}

\begin{abstract}
Motivating sustainable behaviors is increasingly becoming an important topic in the HCI community. While a substantial body of work has focused on the role of peer-pressure through social networks, we argue that the community has largely overlooked the importance of strong social ties and specifically those of family members. We propose the theory of Social Translucence as a theoretical framework for understanding how eco-feedback interfaces can integrate with and support existing communication practices within families. We report on our ethnographic inquiry involving a day reconstruction study followed by in-depth interviewing with 12 families, which took place during a six-month deployment of an eco-feedback interface. Through our study we attempt to inquire into how eco-feedback interfaces: a) raise mutual awareness of family members' consumption behaviors, and b) induce feelings of accountability on individuals regarding their consumption behaviors.
\end{abstract}

\section{Introduction}

Environmental sustainability is an increasingly important global issue. Energy consumption, in particular, is considered to be invisible to millions of users, which makes a prime case of much wastage especially in domestic environments [3]. New technologies in data acquisition and analysis (for instance Non-Intrusive Load Monitoring) provide individuals access to information about their energy consumption that is otherwise difficult to estimate. This has lead to an outburst in interest in the so-called eco-feedback technology, "technology that provides feedback on individual or group behaviors with a goal of reducing environmental impact" [6].

An increasing body of work on eco-feedback technologies stresses the influences that social networks exert on individuals' behaviors. Social influences may contribute towards sustainable behaviors through stimulating competition and providing social incentives [15], supporting public goal commitment [14] and affecting social norms of a culture [7]. In one of the first examples of such work within HCI, Mankoff et al. [12] discussed opportunities and concerns when leveraging the power of social networking sites in influencing individuals' actions. Odom et al. [13] developed and evaluated different eco-visualizations for use in student dormitories. Stepgreen [12] is a social networking site that enables individuals to assess the impact as well as 
receive feedback on their goals with respect to sustainable behaviors. Last, even commercial services such as Google powermeter and Microsoft ohm have adopted social features.

Most existing work has focused on weak social ties, such as neighbors, friends or contacts in online social networking sites. These types of social ties are, however, expected to exert weaker influence on individuals' consumption behaviors when compared with stronger social ties such as family relations [4, 8]. Families often discuss about and encourage pro-environmental behaviours, they maintain a higher awareness of each others' behaviours, and display limited privacy concerns relating to in-house activities and whereabouts, when compared to weaker social ties $[4,8]$.

This paper attempts to contribute to our understanding of how families appropriate eco-feedback interfaces in their daily routines. We employ the framework of Social Translucence as a theoretical lens and we raise two questions: a) how eco-feedback interfaces raise mutual awareness of family members' behaviors, and b) induce feelings of accountability on individuals regarding their consumption behaviors.

\section{Eco-feedback Interfaces as Socially Translucent Systems}

The Theory of Social Translucence [5] argues that motivating desired behavior requires more than making one's behavior visible to his or her social network. It identifies three properties - visibility, awareness, and accountability - of socially translucent systems, systems that support coherent behavior in groups and communities by making participants and their activities visible to one another.

Socially translucent systems first have to make socially significant information, such as one's energy consumption or transport behavior, visible to one's social network. Once this information is visible, people may or may not become aware of this and may act upon it. For instance, they may positively respond to a good act and thus reinforce it or may also become motivated to behave in the same manner. Thirdly, this mutual awareness of each other behaviors eventually results into people feeling more accountable for their actions.

Visibility refers to making one's behavior (e.g. energy consumption) visible to others. We understand visibility in a broad sense, reflecting eco-feedback interfaces' ability to make not only family behaviors, but also the impact of those behaviors, visible among all members of the family. In other words, eco-feedback interfaces need to visualize consumption behaviors within a house but also challenge family misbelieves about what actions may result to energy savings.

According to Social Translucence theory, however, visibility does not guarantee awareness of the information. Contextual aspects such as the location of the ecofeedback interface, as well as the role of different family members when it comes to energy consumption may influence the extent to which families maintain awareness of each others' behaviors. Is the eco-feedback interface accessible to all members of the family, or does the family use one or some of its members as a proxy to the information? Second, mutual awareness of each other's consumption behaviors exists even in the absence of eco-feedback interfaces; the question, then, is: how do ecofeedback interfaces leverage existing communication practices of the family rather than replace them? 
Last, the theory of Social Translucence postulates that accountability of one's consumption behavior is built up through the mutual awareness of each other's actions. Through making consumption behaviors visible to all family members, ecofeedback interfaces are expected to impact the social structure of families. The question raised is: do eco-feedback interfaces participate harmonically in families, and in what ways do they induce feelings of accountability on family members' behaviors?

\section{Study}

\subsection{Participants}

Participants were selected from a list of energy consumption data given by the local electricity company. We selected two buildings that displayed high variation in the energy consumption across individual apartments and invited families to participate in the study through leaving letters in the mailboxes. Out of the 35 apartment occupants that responded to the invitation we selected 30 families who showed interest in the study, and which belonged to the four annual energy consumption levels defined by the company. Two out of the 30 families refused to participate in the study when the system was about to be installed, as they argued it would not be safe to have it around their children. The current study presents findings from our interviews with 12 of these 28 families. Families' residence in those apartments ranged from 2 to 11 years $(\mu=6.25, s d=2.59)$. Parents' age ranged from 30 to 48 years for the fathers $(\mu=37.83$, $\mathrm{SD}=5.75), 29$ to 46 years old for the mothers $(\mu=35.25, \mathrm{SD}=5.78) .10$ out of the 12 families had children. Three children were of age $0-2$, four of age $4-5$, six of age $7-11$, three of age 13-15, and one of age 26 .
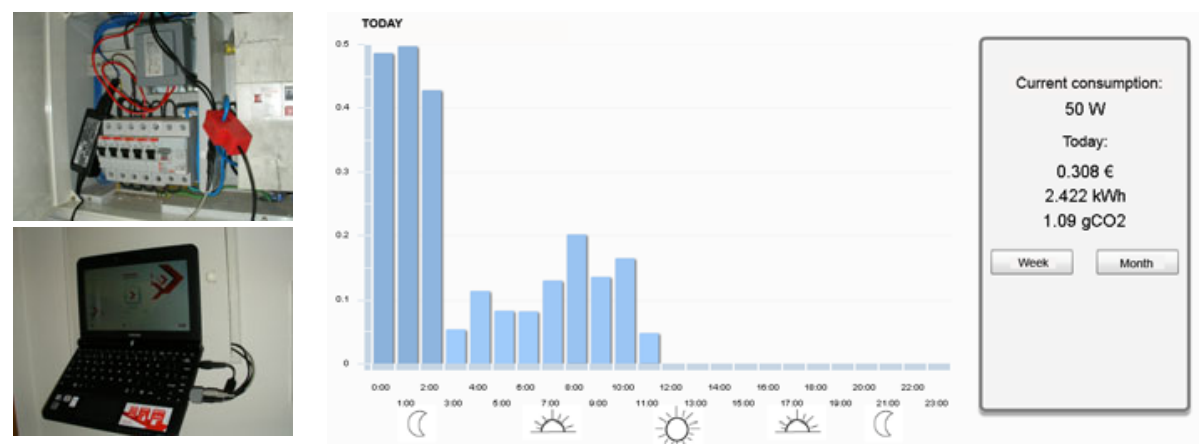

Fig. 1. (left) the powermeter consisted of a netbook and an ADC converter, next to the mains fuse box which in all participants' apartments was located in the main corridor (right). The ecofeedback interface presented information relating to the household's overall consumption per day, week or month, and in terms of $\mathrm{KWh}$, cost and $\mathrm{CO}_{2}$ emissions. 


\subsection{Eco-feedback as Technology Probe}

To sensitize families on energy consumption we introduced a technology probe [9], an energy meter with a simple eco-feedback interface. Our interest was to understand how this system would probe discussion within the family and how families would appropriate it within their daily routines. The energy meter consisted of a netbook and an ADC converter for measuring household energy consumption and inputting it to the netbook through the microphone input. The netbook was installed next to the mains fuse box, which in all participants' apartments was located in the main corridor, a place that we judged appropriate for a public display (see figure 1). The ecofeedback interface presented information relating to the household's overall consumption per day, week or month, and in terms of $\mathrm{KWh}$, cost, and $\mathrm{CO}_{2}$ emissions (see figure 1). The interface was built in Adobe Flex while all computations and handling of user events (i.e. mouse activity, recording people either passing by or standing in front of the netbook) were performed in Processing.

\subsection{Method}

The study consisted of two parts: a) a 1-day diary of all members of the family followed by b) interviews the day after. Using the Day Reconstruction Method (DRM) [10], we asked all family members to list, in a chronological order, the activities they performed while being within the house during the reported day. For each activity they provided a brief name and start and end time. Following the complete reconstruction of all daily activities, participants were asked to provide more detailed information for each activity. This information was: a) electrical devices that were directly or indirectly used in the activity, b) all family members' locations (kitchen, living room, bedroom, outside house, other part of house), and c) subjective ratings on the three social translucence dimensions, namely visibility, awareness and accountability using 7-point likert scales.

Interviews, the day after, took place with all family members present as we wanted to understand how family members would interact when addressing energy consumption issues. Interviews consisted of three parts. Firstly, in a warm-up discussion we probed for general information relating to the family's daily routine, their concerns and their expenses with utilities. Secondly, each member of the family was asked to go through the diary and select one or two activities that they would like to discuss. For each activity we asked them to reminisce the context and motivations for the performed activities and probed for discussion between her and other members of the family on whether and how this activity could be improved with respect to energy consumption. Thirdly, we conducted a contextual inquiry in the usage of the behavior meter and its implication on their behaviors and concluded with a summary of the most substantial insights while asking how the reported day (in the diary study) is different from a typical family day. Qualitative data were analyzed using Affinity Diagrams [1]. Individual statements were printed and posted to a wall. They were then clustered in hierarchical themes followed by labeling the themes. This was an iterative process performed by the first two authors. 


\section{Findings}

Similarly to Broms et al. [2] and Karapanos et al. [11], we observed two phases in families' interactions with the eco-feedback interface: an initial orientation phase that lasted approximately 4 weeks, and after which we saw a decline of about $40 \%$ in users' interactions, followed by an incorporation phase which signified, first, the loss of the powermeter's novelty, and secondly its appropriation in families' daily routines. In the remainder of the paper we describe our findings with regard to our two research questions:

\subsection{How Do Eco-feedback Interfaces Raise Mutual Awareness of Family Members' Behaviors?}

Our probe revealed to make consumption behaviors more visible to the family either through making these more transparent, bringing them to debate or by challenging their performance when conducting them.

Through supporting peripheral awareness. During the incorporation phase the powermeter was primarily used for reassurance purposes, i.e. knowing that everything is as one would expect them to find. The mains fuse box where the powermeter was placed was often located in the main corridor of the apartment and next to its main entrance. Glancing at the powermeter provided peripheral awareness of each others' behaviors both in terms of attention resources and time. Individuals could infer the devices being used at a single point in time only based on the overall disaggregate - household consumption. This often cued an inference about others family members' behaviors such us in the following example: "I saw high consumption and went around to see that the fridge's door was open ... they always forget to close it properly!" (family 7 Father, ref 1). In other cases, the powermeter allowed absent family members to infer household activities on their arrival: "One day I used the dryer to dry some clothes... [my husband] arrived home and asked what I had used between those hours as the consumption was 3 times higher. I said nothing much and then I remembered I had used the dryer" (family 4 Mother, ref1).

Through cueing discussion. We were surprised to observe that only a limited set of cues (such as disaggregate household consumption per hour) was enough to allow rich inferences about household activities. This was possible partly because families do maintain awareness of each other's activities as one father shared with us, "we must coordinate all our tasks, so it is very rare that either she or I have any consumption that the other doesn't know what it is" (family 19, Father, ref7). But even more importantly, we found that, when background information was not sufficient to allow inferences about who and what consumed energy, the powermeter provided cues for discussion among family members as in the previous example of family 4 . We noticed that depending on the type of the activity, families displayed different co-presence patterns (operationalized in terms of number of members collocated in the same room as well as number of members outside the house), with high-consuming activities like doing the laundry or ironing often being solitary (see table 1). Families' activities seem to also have a different distribution across time during weekdays and during weekends (see Figure 2). For instance, activities such as 
personal care, cooking and having a meal happen around the same time during weekdays but are more dispersed during weekends. TV watching was a constant activity throughout both weekdays and weekends. Activities integrated in leisure time were more prone to happen during the weekend.

Through supporting arguments with data. Families revealed to hold misbelieves about each others' consumption behaviors and often conflicts arose, e.g. "he constantly switches on the TV whenever he walks around the house, even if he's not there watching, in the kitchen, in the bedroom, in the living room..." (family 7, Mother, ref3). The powermeter gave family members the ability to support their arguments with data, as in one case where children complained about the fathers' use of his personal computer in response to his criticism on their use of console games. In other cases, family members used energy cues to infer behavior, such as when parents observed that their children spent too much time with console games, or the presence of the maid: "on Mondays the maid comes and vacuums, cleans, irons as we checked in the computer [powermeter] that day is an energy peak for us" (family 28, Father, ref1). In fact, family members rated activities higher in terms of energy consumption when these meant using high perceived consuming devices (cooking, and laundry) or when they would spend a considerable amount of time performing these activities (console games) (see table1).

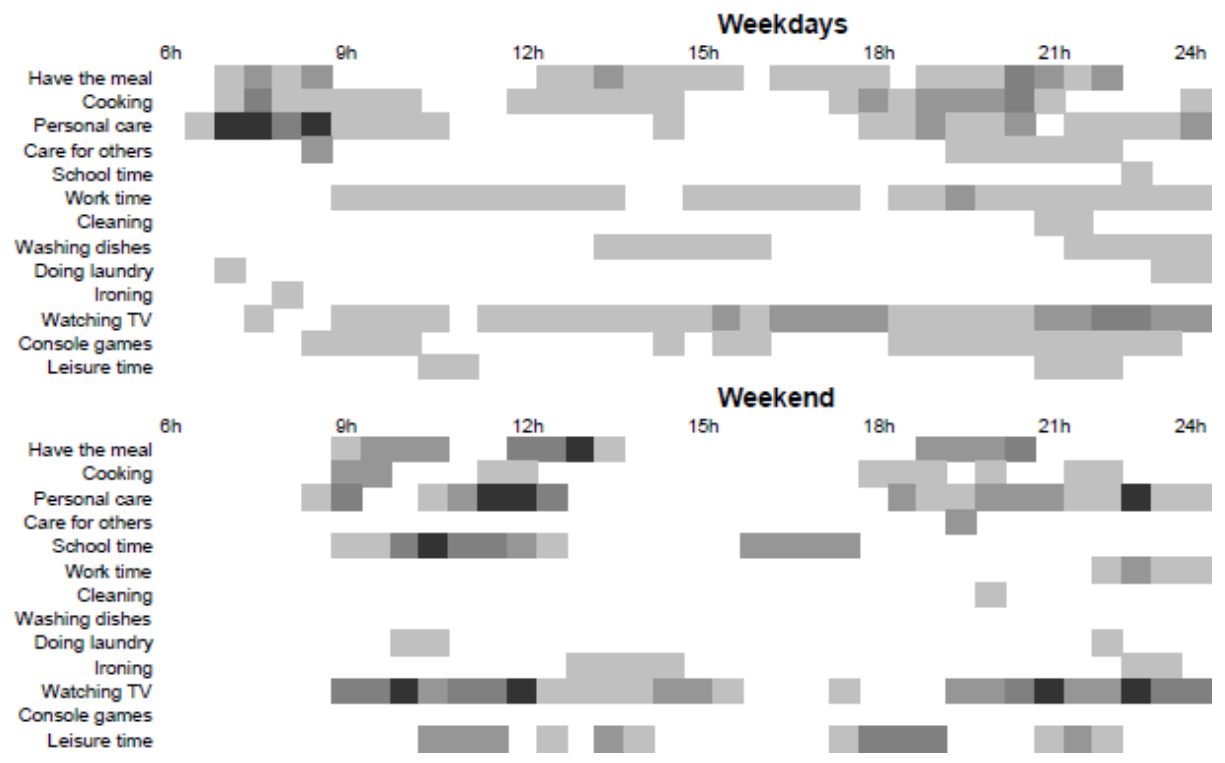

Fig. 2. Distribution of family activities over time during weekdays and weekends

\subsection{How Do Eco-feedback Interfaces Induce Feelings of Accountability on Individuals Regarding Their Consumption Behaviors?}

As it became obvious in the previous section, the powermeter did not provide any radically new information to the family, but instead, it came to enhance the presence 
of consumption information: "After seeing how much using the dryer during the day costs, I am more aware and I will avoid using it. One thing is getting the bill at the end of the month and I see numbers, the other is seeing it there on the screen" (family 4 Mother, refl).

Through leveraging families' existing means. Families have their own means for inducing accountability in individuals' consumption behaviors, such as commenting on others' behaviors, adapting one's own behavior to set the example, leaving subtle messages (e.g. a parent placing environmental magazines at a visible location), or even employing creative ways to do so, e.g. "I use some tape in the switch so they don't use it every time they come" (family 26, Mother, ref 1).

The powermeter leveraged those means through enhancing the presence of costs and environmental impact of energy consumption, both in making it present throughout the whole month (in contrast to the monthly bill) but also in making it accessible to all members of the family, e.g. "They only know [how much it costs] when we complain about the bill, when it is too high" (family 4 Mother, ref 1). For instance, parents would use energy consumption data in educating their children on pro-environmental behavior and expense management: "I had to explain to her (to the youngest daughter) why we need to pay it (electricity) (...) sometimes she wants to stay more time in the water and I tell her we need to save water and gas (family 26 Mother, ref 4). In other cases the powermeter supported more frequent and datagrounded reflection on the family's energy expenses: "I talk to my husband... it is just to keep track of it, we mostly talk about it we don't write it down" (family 5 Mother, ref 1).

Table 1. Number of family members collocated (in the same room), number of family members being outside the house, perceptions of energy consumption and perceived accountability for 13 activity categories. Mean values (standard deviations).

\begin{tabular}{lllll}
\hline Activities & No Collocated & No Outside & Energy & Accountability \\
Have the meal & $2,64(1,32)$ & $0,42(0,77)$ & $4,36(3,10)$ & $4,95(2,42)$ \\
Cooking & $1,50(0,74)$ & $0,53(0,88)$ & $5,61(4,22)$ & $4,43(2,85)$ \\
Personal care & $1,83(1,17)$ & $0,19(0,49)$ & $2,57(2,89)$ & $4,66(2,51)$ \\
Care for others & $2,50(0,85)$ & $0,60(0,84)$ & $2,70(3,27)$ & $2,60(3,21)$ \\
School time & $2,25(1,49)$ & $1,00(0,92)$ & $1,75(0,89)$ & $4,50(0,71)$ \\
Work time & $1,73(1,32)$ & $1,20(1,15)$ & $4,33(2,61)$ & $4,87(2,77)$ \\
Cleaning & $1,57(0,79)$ & $0,14(0,38)$ & $3,86(2,03)$ & $3,57(3,41)$ \\
Washing dishes & $2,50(0,71)$ & $1,50(2,12)$ & $3,50(2,12)$ & $4,50(0,71)$ \\
Doing laundry & $1,16(0,41)$ & $0,16(0,41)$ & $5,00(3,69)$ & $6,00(1,09)$ \\
Ironing & $1,00(0)$ & $0,33(0,58)$ & $4,33(1,53)$ & $6,66(0,58)$ \\
Watching TV & $2,32(1,25)$ & $0,61(1,08)$ & $3,81(1,66)$ & $4,64(2,18)$ \\
Console games & $1,33(0,52)$ & $1,50(1,64)$ & $4,50(0,55)$ & $1,00(0)$ \\
Leisure time & $1,77(0,83)$ & $0,85(0,89)$ & $3,15(1,95)$ & $4,00(1,53)$ \\
\hline
\end{tabular}

Through enforcing transparency - I know that you know. Surprisingly, participants' ratings on accountability over the different reported activities in the day reconstruction study displayed a low correlation with ratings of perceived energy consumption (Pearson's $\mathrm{r}=0.12, \mathrm{n}=240, \mathrm{p}=0.05$ ). Contrary, perceptions of accountability correlated with perceptions of awareness suggesting that one feels more accountable when other family members know about his or her energy 
consumption (Pearson's $\mathrm{r}=0.59, \mathrm{n}=240, \mathrm{p}<0.01$ ). Activities such as having a meal or watching TV (see table 1), which were largely shared activities, displayed higher ratings of accountability. We found that the powermeter changed the way families communicated about consumption behaviors. Firstly, it motivated all members to share their knowledge when inferring what activities caused particular consumption levels as it supported a common family goal. Secondly, through raising mutual awareness of each other behaviors, it induced perceptions of accountability both during engaging with energy consuming behaviors as well as during reasoning and discussing over individuals' behaviors with family members.

\section{Conclusion}

This paper proposed the theory Social Translucence as a framework for understanding how eco-feedback interfaces raise mutual awareness of, and, secondly, induce feelings of accountability on individuals' consumption behaviors. We reported on our initial interviews with 12 families during our 6-month deployment of a simple ecofeedback interface. Our future work will attempt to further inquire into family dynamics using different forms of probing and will attempt to draw implications for the design of eco-feedback interfaces.

\section{References}

1. Beyer, H., Holtzblatt, K.: Contextual design: defining customer-centered systems. Morgan Kaufmann Pub., San Francisco (1998)

2. Broms, L., Katzeff, C., Bang, M., Nyblom, A., Hjelm, S.I., Ehrnberger, K.: Coffee maker patterns and the design of energy feedback artefacts. In: Proceedings of the 8th ACM Conference on Designing Interactive Systems, pp. 93-102 (2010)

3. Darby, S.: The effectiveness of feedback on energy consumption. Environmental Change Institute, University of Oxford (2006)

4. Edgerton, E., McKechnie, J., Dunleavy, K.: Behavioral determinants of household participation in a home composting scheme. Env. and Beh. 41(2) (2009)

5. Erickson, T., Kellogg, W.A.: Social Translucence: An Approach to Designing Systems that Support Social Processes. ACM TOCHI 7(1), 59-83 (2000)

6. Froehlich, J.: Promoting energy efficient behaviors in the home through feedback: The role of human-computer interaction. In: HCIC 2009 Winter Workshop Boaster Paper (2009)

7. Goldstein, N., Cialdini, R., Griskevicius, R.: A Room with a Viewpoint: Using Social Norms to Motivate Environmental Conservation in Hotels. J. Consumer Research 35 (2008)

8. Haeffner, M., Casalegno, F.: How Does a Visual Monitoring System Foster Sustainable Behavior? Instructional Technology, 27-35 (2009)

9. Hutchinson, H., Mackay, W., Westerlund, B., Bederson, B.B., Druin, A., Plaisant, C., Beaudouin-Lafon, M., Conversy, S., Evans, H., Hansen, H., et al.: Technology probes: inspiring design for and with families. In: CHI 2003, pp. 17-24 (2003)

10. Kahneman, D., Krueger, A.B., Schkade, D.A., Schwarz, N., Stone, A.A.: A survey method for characterizing daily life experience: The day reconstruction method. Science 306(5702), 1776-1880 (2004) 
11. Karapanos, E., Zimmerman, J., Forlizzi, J., Martens, J.-B.: User Experience Over Time: An Initial Framework. In: Proceedings of the 27th International Conference on Human Factors in Computing Systems, CHI 2009, pp. 729-738. ACM, New York (2009)

12. Mankoff, J., Fussell, S.R., Dillahunt, T., Glaves, R., Grevet, C., Johnson, M., Matthews, D., Matthews, H.S., McGuire, R., Thompson, R., et al.: StepGreen. org: Increasing energy saving behaviors via social networks. In: Proceedings Fourth International AAAI Conference on Weblogs and Social Media, pp. 23-25 (2009)

13. Odom, W., Pierce, J., Roedl, D.: Social Incentive \& Eco-Visualization Displays: Toward Persuading Greater Change in Dormitory Communities. In: Workshop Proc. of OZCHI, p. 8 (2008)

14. Pallak, M.S., Cummings, W.: Commitment and voluntary energy conservation. Personality and Social Psychology Bulletin 2(1), 27-30 (1976)

15. Petersen, J.E., Shunturov, V., Janda, K., Platt, G., Weinberger, K.: Dormitory residents reduce electricity consumption when exposed to real-time visual feedback and incentives. International Journal of Sustainability in Higher Education 8(1), 16-33 (2007) 